\title{
Debates on Down Syndrome Screening in Iran
}

\author{
Ali Najafi ${ }^{1}$ and Seyed Mohammad Akrami ${ }^{1{ }^{*}}$ \\ ${ }^{1}$ Department of Medical Genetics, Tehran University of Medical Sciences, Tehran, Iran \\ "Corresponding author: MD, PhD of Medical Genetics, Associate Professor of Department of Medical Genetics, Tehran University of Medical Sciences, 1417613151, Tehran, Iran. \\ Email:akramism@tums.ac.ir
}

Received 2021 September 04; Revised 2021 October 03; Accepted 2021 October 17.

Keywords: Down Syndrome, Prenatal Diagnosis, Induced Abortion

\section{Dear editor,}

Recently, fetal aneuploidy screening, also wrongly mentioned as Down syndrome screening, and legal abortion have raised attentions and been a hot topic in Iran. The debates have strongly affected the "population youth and family support" bill of the Joint Commission of the Islamic Parliament of Iran which is going to severely restrict prenatal screening and legal abortion. So, we have discussed facts about prenatal screening in Iran and other countries in a paper entitled review of prenatal aneuploidy screening uptake rate and trends in Iran, and developed countries in the present journal, and here discuss about the related misinformation in recent debates.

Previously, there was a legislation approved by the parliament about therapeutic abortion in 2005 which allowed termination of pregnancies that threatened the mother's life, or fetuses with major anomalies or diseases that would cause many sufferings for the mother. Prenatal screening had started even before this legislation but it was since 2013 that a governmental guideline about screening chromosomal aneuploidies was released with the Ministry of Health, which had periodic revisions, by the last version belonging to 2020 .

Screening critics in Iran have widely reported that the uptake rate of Down syndrome screening (including Edward and Patau syndrome) in developed countries is about $30 \%$ but in Iran, it is $94.5 \%$ (from a sample size of 720 women) (1). The question is whether it is possible to claim that the screening uptake rate in developed countries for the screening of Down syndrome is about $30 \%$ based solely on the participation of pregnant women in the Netherlands in first trimester combined (FTC) test with the data of a decade ago? Different screening methods can be used in one country. So, we have to take all of them into account in our calculation. For example, the combined uptake rate of FTC test and non-invasive prenatal screening (NIPT) in
2019 in the Netherlands was 48.3\% (2); to be compared with about $30 \%$ stated by the critics.

By reviewing prenatal screening uptake rates in developed countries, it is obvious that on average this rate is more than twice the amount mentioned by the critics and this rate has been increasing since the introduction of these tests. The policy of most of the developed countries is to recommend screening to all pregnant women.

Critics say that the rate of false-positive results and the rate of performed invasive diagnostic tests in the prenatal screenings done with the Iranian health centers (in fact in these centers, no invasive test is performed nor referred to be done) is high and claim the national average of false-positive rate of prenatal screening and the rate of performed invasive diagnostic tests is $12.5 \%$ and $4.4 \%$, respectively (3). These and many other statistics about the screening situation in Iran that are being cited by screening critics were presented by a retrospective study that collected data by telephone interview from 2096 mothers or their husbands (1). However, we should know that in the study they have considered anyone performing a secondary (supplementary) test, as being the result of positive screening in the primary prenatal screening test, while many of these cases were not positive with the national guidelines. The correct execution of the current national screening protocols leads to a false positive rate (FPR) of $5 \%$, which is considered a standard value in terms of global statistics (4). Also, the claimed rate of invasive tests in Iran is within the global norms.

It could be much better for the critics to use laboratory statistics instead of conducting a telephone interview that can have high rates of error. The critics have also compared the goals mentioned in the foreign guidelines with the values obtained from their telephone interviews. There are some problems in this regard:

1- The number of performed supplementary (sec- 
ondary) tests is not equal with the number of false positive results from prenatal screening tests. Therefore, it is wrong to consider the number of supplementary tests or the number of recommendations to do these tests or even the number of pregnant mothers perceiving that they need to perform these tests, with the actual FPR of screening tests.

2-As the study was retrospective and with a telephone interview, factors such as problems with random sampling or misconceptions of the mothers or their husbands about the outcome of screening or the direction of the interview.

3- In the study, more than $30 \%$ of the people did not participate in the interview, which can affect the results obtained. In such studies, families who have faced problems within the screening process may be more likely to participate in these interviews and bias the results.

4- Basically, NIPT was not included in the national Down syndrome screening protocol in 2017, and on the other hand, integrated serum test was recommended for pregnant women who did not have access to NT (nuchal translucency) ultrasound. It is not clear whether in that study, participants in the two-stage integrated serum test were considered within false positive results. It seems that in the mentioned study, due to the weakness in understanding different types of screening and diagnostic tests, the FPR was stated higher.

5- To calculate the FPR, this number had to be calculated for each of the different screening methods, so potential problem in any of them could be further analyzed individually.

6- Goals of guidelines can differ with actual state of screening.

Also, regarding the claim of high rate of invasive diagnostic tests in the health centers and the whole country, in the new Down syndrome screening guideline (version 2020), the Genetics Office of The Ministry of Health has changed the cutoff for recommending invasive sampling from $1 / 250$ to $1 / 10$ for most cases. This would greatly reduce the rate of amniocentesis and CVS. So, potential samplingrelated abortions will come close to zero.

Regarding the rate of abortion in the second trimester, which has been attributed to screening and said that illegal abortion of about 20,000 to 33,000 fetuses per year is due to high-risk screening results and inability of mothers to perform additional tests due to high cost of diagnostic tests. The number comes from considering that we only have $0.5 \%$ spontaneous abortion rate in the second trimester and any extra numbers belongs to the abovementioned induced abortions. In fact, they were totally wrong about the spontaneous abortion rate in second trimester which can vary from 1 to even $5 \%$ and their observed second trimester abortion rate $(2.6-2.7 \%)$ is within this range. Also, differences between races and ethnicities in spontaneous abortion rate must be considered.

Another important point is about the percentage mentioned by the critics for abortions caused by invasive tests, which they considered it between 0.5 to $2 \%$ and used in their calculation. To cite $2 \%$, they referred to a small survey in Scotland based on only 130 CVS samples, wherein reasons other than fetal anomalies were not considered in their calculation (5). On the other hand, we have a metaanalysis on pregnancy loss related to amniocentesis and CVS in 2019 that shows the risk is 0.0012 and -0.0011 , respectively (6).

The critics claim that the cost of Down syndrome screening is 121 times the cost of having no screening in Iran. To do this, they first calculated the birth costs of each person with Down syndrome with US standards, and when converting this amount into the equivalent of services in Iran, used the nominal GDP per capita of the two countries. On the other hand, used gross national income (GNI) per capita, PPP (current international \$) to calculate the loss caused by abortion of any healthy fetuses. They multiplied the GNI number by 50 for 50 working years considered for each lost healthy child but they have used higher GNI than the value in their reference from World Bank $(\$ 1,050,500$ vs. $\$ 728,000$ ). GNI and GDP values per capita don't differ a lot but they both had to be in PPP form. Using nominal GPD per capita understate the costs of taking care of the affected children by about 3-fold. In addition, considering that the rate of the claimed induced abortion lacks sufficient scientific evidence and the rate of procedure-related miscarriage considered by them is very high, their calculations aren't realistic. Taking into account these weaknesses in the calculation, certainly the ratio of the costs of screening vs. no screening is very different and calculation by the Ministry of Health on different screening protocols have shown that no screening strategy will cost 6 to 7 times more.

These screenings are not just for Down syndrome and they are only a part of the embryos with disabilities that are identified in the procedure of the tests and as a result, the cumulative costs are higher. These costs are apart from their intense psychological and emotional burden on families and society. The effect of increased divorce rate, reduced father's working hours, and reduced possibility of mothers working out of home, as well as reduced desire to have more children in families with disabled children also has to be considered in any national assessment and decision making about prenatal screening and legal abortion. 


\section{Footnotes}

Authors' Contribution: S.M.A. developed the original idea, A.N. and S.M.A. gathered and analyzed relevant data, A.N. abstracted and wrote the manuscript, S.M.A. revised the manuscript.

Conflict of Interests: No conflict of interests.

Funding/Support: It was not declared by the authors.

\section{References}

1. Zeinalloo M. [Cost analysis of Down Syndrome screening during pregnancy in the health network in 2018 and presentation of a proposed program].Tehran Uni Med Sci. 2020. Persian.
2. Liefers J, Atsma F. [Monitor 2019, Prenatal screening for Down syndrome, Edward, and Patau syndrome and the Structural Ultrasound Examination]. IQ healthcare. 2020. Dutch.

3. Shariati M. [Down Syndrome Screening Challenges in Pregnancy Care; Opportunities and threats]. Iranian Students' News Agency. 2021. Persian.

4. Hancock S, Johansen Taber K, Goldberg JD. Fetal screening and whole genome sequencing: where are the limits? Expert Rev Mol Diagn. 2021;21(5):433-5. doi: 10.1080/14737159.2021.1919087. [PubMed: 33866903].

5. Tydeman G, Bremner C. Amniocentesis and Chorionic Villus Sampling in Scotland: An audit of techniques and outcomes of all procedures over one year in Scotland, 1 May 2008 - 30 April 2009. Healthcare Improvment Scotland; 2010.

6. Salomon LJ, Sotiriadis A, Wulff CB, Odibo A, Akolekar R. Risk of miscarriage following amniocentesis or chorionic villus sampling: systematic review of literature and updated meta-analysis. Ultrasound Obstet Gynecol. 2019;54(4):442-51. doi: 10.1002/uog.20353. [PubMed: 31124209]. 